\title{
Randomised controlled trial of a home- based physical activity intervention in breast cancer survivors
}

\author{
Ian M. Lahart ${ }^{1 *}$, George S. Metsios ${ }^{1}$, Alan M. Nevill', George D. Kitas ${ }^{1,3}$ and Amtul R. Carmichael ${ }^{2}$
}

\begin{abstract}
Background: To improve adherence to physical activity (PA), behavioural support in the form of behavioural change counselling may be necessary. However, limited evidence of the effectiveness of home-based PA combined with counselling in breast cancer patients exists. The aim of this current randomised controlled trial with a parallel group design was to evaluate the effectiveness of a home-based PA intervention on PA levels, anthropometric measures, health-related quality of life (HRQOL), and blood biomarkers in breast cancer survivors.

Methods: Eighty post-adjuvant therapy invasive breast cancer patients (age $=53.6 \pm 9.4$ years; height $=161.2 \pm 6.8 \mathrm{~cm}$; mass $=68.7 \pm 10.5 \mathrm{~kg}$ ) were randomly allocated to a 6-month home-based PA intervention or usual care. The intervention group received face-to-face and telephone PA counselling aimed at encouraging the achievement of current recommended PA guidelines. All patients were evaluated for our primary outcome, PA (International PA Questionnaire) and secondary outcomes, mass, BMI, body fat \%, HRQoL (Functional assessment of Cancer Therapy-Breast), insulin resistance, triglycerides (TG) and total (TC), high-density lipoprotein (HDL-C) and low-density lipoprotein (LDL-C) cholesterol were assessed at baseline and at 6-months.

Results: On the basis of linear mixed-model analyses adjusted for baseline values performed on 40 patients in each group, total, leisure and vigorous PA significantly increased from baseline to post-intervention in the intervention compared to usual care (between-group differences, 578.5 MET-min $\cdot \mathrm{wk}^{-1}, p=.024,382.2 \mathrm{MET}$-min.wk ${ }^{-1}, p=.010$, and 264.1 MET-min $\cdot w^{-1}, p=.007$, respectively). Both body mass and BMl decreased significantly in the intervention compared to usual care (between-group differences, $-1.6 \mathrm{~kg}, p=.040$, and $-.6 \mathrm{~kg} / \mathrm{m}^{2}, p=.020$, respectively). Of the HRQOL variables, FACT-Breast, Trial Outcome Index, functional wellbeing, and breast cancer subscale improved significantly in the PA group compared to the usual care group (between-group differences, $5.1, p=.024 ; 5.6, p=.001 ; 1.9$ $p=.025$; and 2.8, $p=.007$, respectively). Finally, TC and LDL-C was significantly reduced in the PA group compared to the usual care group (between-group differences, $-.38 \mathrm{mmol} \cdot \mathrm{L}^{-1}, p=.001$; and $-.3 \mathrm{mmol} \cdot \mathrm{L}^{-1}, p=.023$, respectively).
\end{abstract}

Conclusions: We found that home-based PA resulted in significant albeit small to moderate improvements in selfreported PA, mass, BMI, breast cancer specific HRQoL, and TC and LDL-C compared with usual care.

ClinicalTrials.gov identifier: NCT02408107 (March 25, 2015)

Keywords: Breast neoplasms, Physical activity, Randomised controlled trial

\footnotetext{
*Correspondence: i.lahart@wlv.ac.uk

${ }^{1}$ Faculty of Education, Health and Wellbeing, University of Wolverhampton,

Walsall Campus, Gorway Road, Walsall WS1 3BD, UK

Full list of author information is available at the end of the article
} 


\section{Background}

Worldwide, breast cancer is the most frequently diagnosed cancer and the leading cause of cancer death among females [1]. In the UK, female breast cancer has the highest incidence rate of all cancers [2], and is predicted to increase by $44 \%$ up to 2020 [3]. Owing largely to early detection and improved treatment strategies, UK breast cancer mortality rates are falling [4], resulting in the largest prevalence of breast cancer survivors in the UK ever reported.

Due to the prevalence of treatment-related health concerns and increased risk of developing metabolic syndrome, recurrence and cardiovascular disease, breast cancer survivors may require diagnostic, therapeutic, supportive or palliative services for many years postdiagnosis [5-7]. Encouraging breast cancer survivors to adopt a healthy lifestyle post-treatment may reduce the healthcare burden resulting from treatment-related sequelae and improve survival [8]. In particular, higher levels of physical activity (PA) may reduce risk of recurrence and all-cause and breast cancer-related mortality [9-12]. However, PA levels are generally low among breast cancer survivors and many women decrease their PA following diagnosis [13-15]. Therefore, interventions are required to improve the post-diagnosis PA levels of breast cancer survivors.

Randomised controlled trials (RCTs) have found improvements in PA levels, cardiorespiratory fitness, HRQoL, fatigue and weight maintenance in breast cancer survivors participating in PA interventions compared with control groups [16-27]. However, most PA RCTs consist of either entirely or partly facility-based interventions, and therefore, the findings of these trials may not generalise to patients who have limited access to exercise facilities because of transportation, time-related and financial difficulties [22]. In addition, facility-based studies may lack external validity, or real world application, which limits the translation of their findings into practice [28]. To overcome this problem some trials have provided entirely home-based PA interventions $[17,18,20,22,24-27]$. In addition to mitigating transport, time-related and financial difficulties, home-based interventions are also advantageous because they are less expensive than supervised, facility-based interventions and do not require participants to attend classes or maintain a health club membership to sustain PA [22].

For breast cancer survivors to maintain their PA participation during and after the specified intervention period, it is important that they are given behavioural change support [19]. However, only three home-based intervention trials included a specific PA behavioural change support component, consisting of both face-toface counselling and support telephone calls [17, 20, 27]. Although the findings of these home-based PA trials are promising, they had a number of limitations (small sample sizes and short intervention duration of 12 weeks, $[17,20,27]$; postmenopausal women only, $[17,27])$ that limit the generalizability of their results. Therefore, the aim of this current study was to investigate the effects of a pragmatic (i.e. designed to test the effectiveness of an intervention in a broad routine clinical practice, [29]) 6-month home-based PA intervention with counselling on PA levels, weight maintenance, HRQoL, and blood biomarkers in breast cancer survivors.

\section{Methods \\ Participants}

Women attending breast cancer clinics between January 2010 and March 2013 at Russells Hall Hospital (Dudley Group NHS Foundation Trust, UK), were invited to participate. Participants were eligible to participate if they were: 1) females aged 18-72 years, 2) diagnosed with invasive breast cancer (Stage I-III) within two years of enrolment, 3) post-surgery and had no surgery planned for the next six months at least, 4) had fully completed adjuvant therapy (radiotherapy and/or chemotherapy) not including hormonal therapy, 5) no previous malignancy, 6) willing to be randomised 7) and willing to maintain contact with the investigators over the six months. Exclusion criteria included: 1) inability to participate in PA because of severe disability (e.g. severe arthritic conditions), 2) psychiatric illness and 3) vulnerable subjects, such as pregnant women or any other patient where PA was not approved by their oncologist due to the presence of one or more contraindications to exercise in cancer patients [30]. Participants who were physically active at the time of enrolment were not excluded from participation. The study was approved by the Black Country NHS Ethics Committee. All participants provided written consent prior to data collection.

\section{Randomisation}

At a Clinic Trials Unit on a different site, a computer generated random numbers list was used to allocate all participants into intervention or usual care groups (concealed from the primary researcher), and allocate $40 \%$ of participants in each group into a substudy involving cardiorespiratory fitness assessment (data not reported). Patients were allocated to intervention and usual care groups on a 1:1 ratio and were stratified based on adjuvant chemotherapy. Randomisation occurred after participants had completed baseline questionnaires and had a blood sample taken.

\section{Home-based PA intervention}

Following randomisation, patients received an intervention aimed at encouraging the adoption of a more physically active lifestyle. Participants received a face-to-face 
consultation, followed by a support telephone call at the end of months one, two and three (i.e. a total of 3 telephone calls). During each of the last two months (4 and 5) patients received a mailed PA reminder leaflets encouraging their participation in home-based physical activity. The intervention was based on the findings from previous research [31, 32], which suggested that breast cancer survivors had strong preferences for the receipt of face-to-face counselling from exercise professionals and for moderate-intensity PA at home and/or outdoors.

Face-to-face consultations were conducted by the primary researcher immediately after initial baseline measurements and were based on the four core motivational interviewing principles: expressing empathy, developing discrepancy, rolling with resistance and supporting selfefficacy [33, 34]. To ensure consistency in intervention delivery, a semi-structured motivational interviewingbased intervention protocol was developed to guide intervention delivery. The topics covered in the 30-45 $\mathrm{min}$ consultation were similar to other trials that incorporated a PA counselling component [17, 20, 22, 27], including: current PA behaviour, decision balance exercise; benefits of PA in general and specific to breast cancer survivors; perceived barriers; prompts to seek social support, goal setting, types and intensities of PA (e.g. explanation of light, moderate and vigorous PA with examples specific to participants, such as, taking a brisk walk so that you are mildly breathless but can still hold a conversation); safety advice; and basic lifestyle information (e.g. basic dietary information, portion size, fat intake, smoking, and hydration in generally and during activity).

The focus of the follow-up phone calls (end of months 1-3) was to prevent relapse back to inactivity and/or improve maintenance of PA (accumulate $30 \mathrm{~min}$ of moderate-intensity PA on 3-5 days/week), and covered topics similar to the face-to-face consultation. Calls lasted approximately 15-20 $\mathrm{min}$ and were guided by standardised phone call scripts. Participants were encouraged to telephone the research team should they encounter any problems or relapse in their efforts to increase their PA. Therefore, our intervention represented a pragmatic step down approach (i.e. from in-person sessions to telephone calls to postcard prompts), that could feasibly be employed by cancer care nurses in routine clinical practice.

The initial goal of the intervention (months 1-3) was for participants to progress towards accumulating 30 min of moderate intensity PA on three to five days per week. During months three to six, the intervention participants were encouraged to work towards accumulating at least $30 \mathrm{~min}$ of moderate-intensity PA on five to seven days per week in broad agreement with current public health guidelines [35]. If participants were already achieving this on trial entry they were, as a minimum, actively encouraged to maintain their level of PA. Participants were encouraged to first focus on the frequency of their PA and then duration.

Participants were given a PA pack consisting of an information booklet and a DVD (previously developed by Breast Cancer Care) that provided further information of topics such as exercising safety, exercise intensity, dealing with fatigue and exercising with lymphedema. Information about local physical activity opportunities was also provided, including an exercise initiative run in local parks. During the intervention period, participants were encouraged, but not required to keep PA diaries to check against whether they were achieving $150 \mathrm{~min}$ of moderate-vigorous PA over each week. Participants were advised to refrain from activity if they experienced any problems relating to the PA intervention (e.g. chest pain or developed a joint problem). If these circumstances occurred, patients would have been advised to contact the clinical team, and the clinician of the research team would have made a clinical decision based on the contraindications and precautions to PA for patients with cancer as to whether the patient refrained from PA temporarily or withdrew from the intervention [30].

\section{Usual care group}

Participants randomised to the usual care arm received standard information regarding PA (i.e. current recommended PA guidelines), as provided to all breast cancer patients treated at the site. Usual care group participants were instructed to maintain their current lifestyle. After completion of the intervention participants in the usual care group were encouraged to adopt a more physically active lifestyle and were given the same guidance and physical activity pack as the intervention group.

\section{Outcomes}

After randomisation, all participants' had their height, mass and body composition measured and completed a demographics questionnaire, interview-administered long form International PA Questionnaire (IPAQ), Functional Assessment of Cancer Therapy-Breast (FACT-B) questionnaire and blood collection. The primary outcome of the current study was total PA (MET-min. $\mathrm{wk}^{-1}$ ). Body composition (body fat \%) was assessed after a 12-h water-only fast by bioelectrical impedance analysis (BIA) using a Tanita BC-418 MA Segmental Body Composition Analyser, which incorporates eight tactile electrode (Tanita Corporation, Tokyo, Japan). The specific device has a standard error of $<3 \%$ when standard procedures are followed [36]. Body mass was also measured via the Tanita analyser and was recorded to the nearest $1 \mathrm{~kg}$. BMI $(\mathrm{kg} /$ $\mathrm{m}^{2}$ ) was calculated on the basis of measured height and mass. Standing height was measured without shoes to the 
nearest $.5 \mathrm{~cm}$ on a portable stadiometer (Seca 214 Road Rod, Seca gmbh \& co. kg., Hamburg, Germany).

Participants completed the validated IPAQ-long form questionnaire, which assesses the duration (number of days $\times$ hours/min per day) that an individual has engaged in walking, moderate, and vigorous PA across four domains (occupational, active transportation, domestic, and leisure) over the past seven days [37]. PA data were then used to calculate the metabolic equivalent (MET)based IPAQ score by weighting each type of activity by its MET energy requirement $(3.3 \times$ walking duration; $4 \times$ moderate PA duration; $8 \times$ vigorous PA duration). Data were summed across activity domains to produce a weighted estimate of total PA (primary outcome) from all reported activities per week (MET-min $\cdot \mathrm{wk}^{-1}$ ), as well as subtotal of activity for each of the four domains, as well as walking, moderate and vigorous PA. The IPAQ allows individuals to be categorised into those who are meeting the current recommended PA guidelines (high and moderate PA categories) and those who are not (low PA category) [35]. Categorisation is based on the following algorithm: 1) high: vigorous PA on $\geq 3$ days and accumulating $\geq 1500 \mathrm{MET}$-min $\cdot \mathrm{wk}^{-1}$, or $\geq 7$ days of any PA accumulating $\geq 3000$ MET-min $\cdot \mathrm{wk}^{-1}$; 2) moderate: vigorous PA on $\geq 3$ days for $\geq 20 \mathrm{~min} /$ day, or moderate $\mathrm{PA} /$ walking on $\geq 5$ days for $\geq 30 \mathrm{~min} /$ day, or $\geq 5$ days of any combination of PA accumulating $\geq 600 \mathrm{MET}-\mathrm{min} \cdot \mathrm{wk}^{-1}$; and 3) low: any combination of PA accumulating $<600$ MET$\mathrm{min} \cdot \mathrm{wk}^{-1}$. The IPAQ has good reliability (Spearman's rho $=.8$ ) and moderate concurrent validity (Spearman's rho $=0.33$ ) when compared to accelerometer data (Spearman's rho $=.33)$ [37].

FACT-B is a 36-item compilation of questions subdivided into four primary HRQoL domains, including physical well-being (PWB; 7-items), social/family well-being (SWB; 7-items), emotional wellbeing (EWB; 6-items) and functional well-being (FWB; 7-items), and a disease specific domain, the breast cancer subscale (BCS; 9-items) [38]. The four primary HRQoL domains are combined to provide a 27-item general HRQoL assessment (FACT-G). The total FACT-B score is calculated by the sum of FACT-G and breast cancer subscale scores. The Trial Outcome Index (TOI), which provides an efficient summary index of physical/functional outcomes was also calculated as the sum of the PWB, FWB and breast cancer subscale scores. Possible score ranges were $0-36$ for the BCS, 0-104 for the FACT-G, 0-140 for the FACT-B, and 0-92 for the TOI. Higher scores represent better quality of life or less severe symptoms. FACT-B has been validated in the breast cancer setting, with good internal consistency (alpha coefficient $=.9$ ), reliability, patient acceptability and sensitivity to clinically significant change [38]. All assessments were made at baseline and within two weeks of completing the 6-month intervention.

\section{Blood collection and laboratory analysis}

Participants were instructed not to exercise for at least 28-h before blood collection. Blood was collected between 9:00 a.m. and 11:00 a.m. on the same day as other assessments after 12 -h water-only fast. The Vitros ${ }^{\circ} 5$, IFS chemistry system (Ortho Clinical Diagnostics Inc., Rochester, New York, USA) was used to measure all lipid components; however, total cholesterol (TC), high-density lipoprotein (HDL-C), and triglycerides (TG) were measured using multi-layered slides, whereas measurement of lowdensity lipoprotein (LDL-C) required a dual chamber package. Plasma glucose was measured using the VITROS $^{\circ} 5.1$ FS chemistry system (Johnson and Johnson Inc., Langhorne, PA, USA) and the same procedure as with cholesterol (but not LDL-C) was followed. Insulin was estimated from serum stored at $-20{ }^{\circ} \mathrm{C}$. The method of detection is a solid phase two-site chemi-luminescence immunometric assay. The Immunolite 2000 insulin was used on the Immulite 2000 Analyser (Siemens Healthcare Diagnostics, Deerfield, IL, USA). Homeostasis Model Assessment (HOMA) of insulin resistance (IR) was evaluated from fasting glucose and insulin [39].

\section{Sample size calculation and statistical analysis}

Power calculations were based on total PA as the primary outcome. Using a between-group mean (SD) change in self-reported PA of 16.5 (25.1) MET-h.wk ${ }^{-1}$ found in a similar trial [20], we estimated that with at least 36 participants in each group $(N=72)$, the trial would have $80 \%$ power at $p<0.05$. To allow for $10 \%$ attrition we aimed to recruit 80 participants (40 in each group). Continuous variables were expressed as mean $\pm S D$, while categorical data were presented as number of participants and percentages.

We used linear mixed-model analysis to examine the differences in the PA intervention group compared with the usual care group in changes over time from baseline to 6-month follow-up for all continuous outcome measures. Each analysis was adjusted for the baseline value of the outcome to control for between group baseline imbalances. Other covariates, including age, BMI (for non-anthropometric analyses), time since diagnosis (weeks), and time since treatment completion (weeks), were adjusted for but did not influence estimates so were not included. For each analysis, to select the best model, $-2 \log$ likelihood (i.e., maximum likelihood ratio test/deviance test) was used. Compared to a model with first-order, auto-regressive covariance structure for the repeated component (time) and a diagonal error covariance structure for the random effect (group), a model with unstructured variance for the repeated component and a compound symmetry for the random effect provided the best model, in all analyses. We used the intention-to-treat principle. For participants with 
missing data at post-intervention or follow-up, we included all available data under the missing-at-random assumption of the mixed-model analysis.

We performed per-protocol analyses among participants who completed both baseline and post-intervention assessments using a contemporary magnitude-based inferences approach [40]. In this approach, mean effects of the PA intervention and their $90 \%$ confidence limits were estimated with a spreadsheet [41] via the unequal-variances $t$ statistic computed for change scores between baseline and post-intervention in the two groups and adjusted for baseline values of each outcome. Each participant's change score was expressed as a percentage of baseline score via analysis of log-transformed values, to reduce bias arising from non-uniformity of error. For this approach, effect sizes were calculated by dividing the log-transformed mean differences between intervention and usual care groups divided by the pooled logtransformed baseline SD of outcomes. The spreadsheet also computed quantitative and qualitative chances that the true effects were beneficial/positive, trivial, and harmful/decrease when a value for the smallest meaningful change was entered. A Cohen unit of .2 was employed as the smallest meaningful change in outcomes. Where the chance of benefit and harm are both $>5 \%$, the effect is deemed unclear. Qualitative descriptors were then assigned to the quantitative percentile scores as follows: $25-75 \%$ possible, $75-95 \%$ likely, and $>99 \%$ most likely.

Chi-square analysis was planned on IPAQ categorical data but was not possible because greater than $20 \%$ of the expected counts were less than five and some of the expected frequencies were below one. Collapsing the moderate and low categories into one category did not remedy this. Therefore, the PA data were presented as frequencies in those who completed post-intervention assessments. The FACT-B, FACT-G, TOI, and BCS HRQoL variables were categorised based on whether participants experienced a minimum clinically important (based on performance status and pain anchors) increase from baseline to post-intervention [42]. Chi-square analysis was then performed to examine intervention and usual care groups for differences in the number of participants who experienced a minimum clinically important increase in these variables. In the intention to treat analysis, standardized effect sizes were calculated for all outcomes by dividing the adjusted between-group difference of the post-intervention means by the pooled baseline standard deviation. According to Cohen [43], effect sizes <.2 indicate 'no/trivial difference', effect sizes of .2 to .5 indicate 'small differences', effect sizes of .5 to $<.8$ indicate 'moderate differences', and effect sizes $\geq .8$ indicate 'considerable differences'. The level of significance was set at $p<.05$.

\section{Results}

Flow of participants through the trial and recruitment Eighty participants were recruited for this trial between January 2010 and March 2013. Flow of participants through the study is provided in Fig. 1, including number of recruited participants and reasons of dropping out.

\section{Participant characteristics at baseline}

Table 1 provides the baseline characteristics overall and by group assignment. Baseline data were collected from 80 breast cancer survivors $(\mathrm{age}=53.6 \pm 9.4 \mathrm{y}$; height $=$ $161 \pm 6.8 \mathrm{~cm}$; mass $=68.7 \pm 10.5 \mathrm{~kg}$ ). The baseline characteristics of participants in the intervention and usual care groups were overall similar in most demographic (Table 1), anthropometric characteristics (Tables 1 and 2) HRQoL and biomarkers (Table 3), with only a few dissimilarities (e.g. usual care group reported more comorbidities). Those in the usual care group were more physically active compared with the intervention group at baseline (Table 2), which was due mainly to a greater amount of domestic PA. Regarding IPAQ PA categories (Table 1), at baseline $15 \%(n=6)$ more participants were categorised in the high activity category in the usual care group compared with the intervention group. No adverse events were reported during the 6-month intervention period, although one participant in the usual care group dropped out due to PA unrelated-sciatica (see Fig. 1).

\section{PA outcomes}

When adjusted for baseline levels, the intervention resulted in a significant but small increase in total PA compared to the usual care group $(p<.05 ; d=.44)$ (Table 2). In particular, leisure PA and vigorous PA increased significantly and moderately in the intervention compared with the usual care group over the intervention period (both $p \leq .01 ; d \leq .60$ ). The per-protocol, magnitude-based inference (adjusted for baseline levels) analysis revealed the effect of the PA intervention was likely to have been beneficial ( $80 \%$ likelihood of a beneficial effect) on moderate PA despite a non-significant main effect, compared with the usual care (Table 4). However, the effect of the intervention was possibly beneficial on all other PA (50\% likelihood of a beneficial effect), except for a possible negative effect on workbased and active transport.

Regarding categorical PA data, $88 \%(n=7 / 8)$ and $12 \%(n=1 / 8)$ of the participants in the intervention group categorised as low activity at baseline, moved to the moderate and high activity category postintervention, respectively. In the usual care group, $50 \%$ $(n=2 / 4)$ remained in the low activity category while only one participants each moved from low to moderate and high activity categories, post-intervention. 


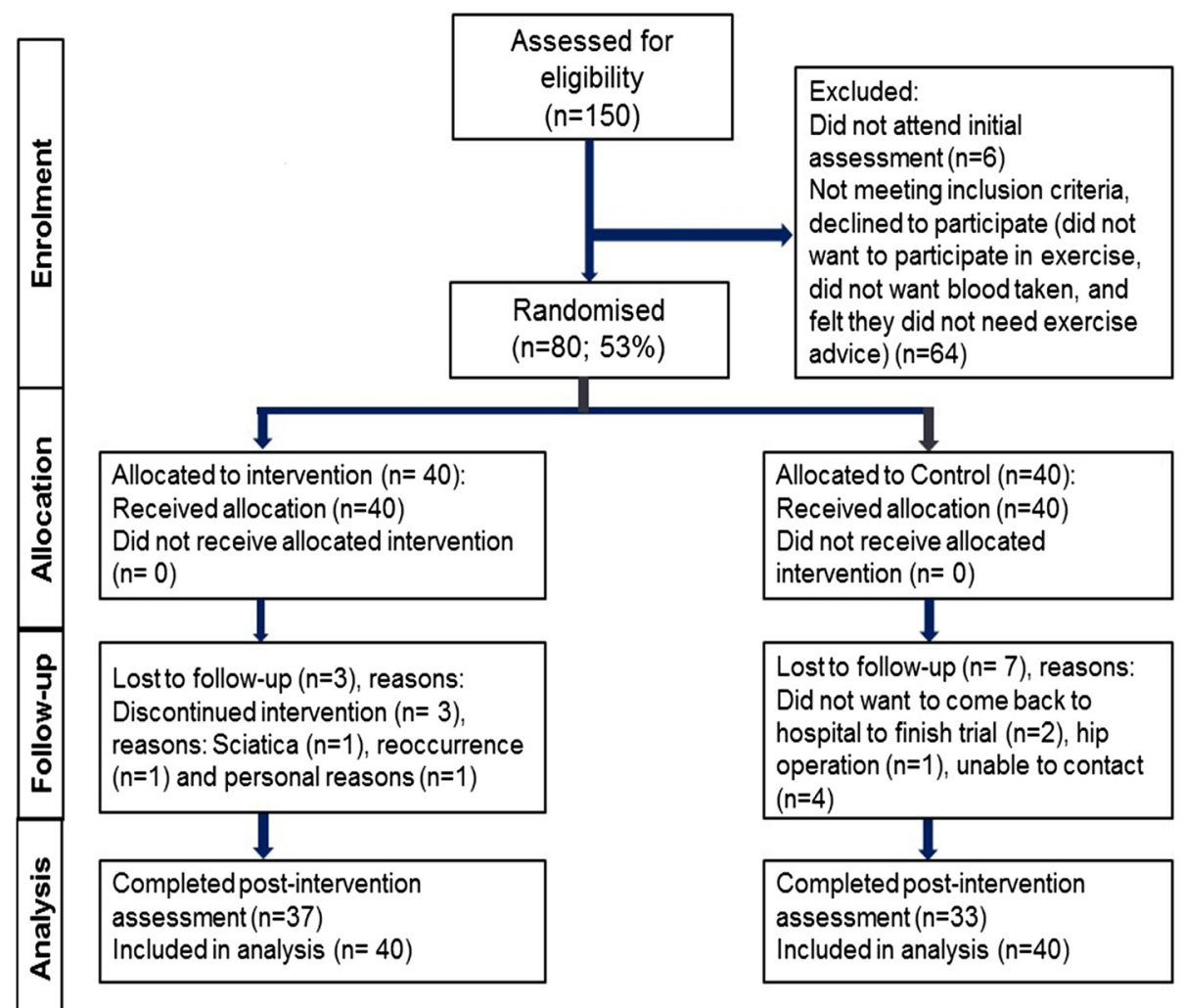

Fig. 1 Flow of participants through the trial

\section{Anthropometric outcomes}

The intervention group experienced trivial but significant decreases in both body mass and BMI from baseline to post-intervention compared with the usual care group (both $p<.05, d<.02$ ). However, no significant change in body fat \% was observed (Table 2).

\section{HRQoL outcomes}

Analyses highlighted a significant but small improvement in FACT-B, TOI, FWB and BCS scores in the PA group compared with the usual care group over the 6month intervention period $(p<.05$ and $d<.50$, respectively). No significant differences between PA and usual care groups were found for any of the other HRQoL variables (Table 3). Magnitude-based inference adjusted analysis of study completers revealed the effect of the PA intervention was only possibly to have been beneficial (80\% likelihood of a beneficial effect) on all HRQoL, compared with the usual care (Table 5).

Chi-square analysis of the FACT-B, FACT-G, TOI, and $\mathrm{BCS}$ variables revealed significant associations between intervention and usual care groups and the number of participants who experienced minimum clinically important increases in TOI, $\chi^{2}(1)=8.34, p=.004$, and BCS, $\chi^{2}(1)=6.19, p=.013$. More than twice as many participants in the intervention group experienced minimum clinically important improvements in BSC and TOI between baseline and post-intervention compared with the usual care group (57 \%, $n=21 / 37$ vs. $27 \%, n=9 / 33$; and $65 \%, n=24 / 37$ vs. $30 \%, n=10 / 33$ ). No significant associations were found between intervention and usual care groups and the number of participants who experienced minimum clinically important changes in FACT-B, $\chi^{2}(1)=1.67, p=.23$, and FACT-G, $\chi^{2}(1)=0.31, p=.63$.

\section{Blood biomarker outcomes}

We found significant but small reductions in $\mathrm{TC}$ and LDL-C concentrations in the PA group compared with the usual care group over the 6-month intervention period ( $p<.05$ and $p<.01$, respectively, and $d<.05)$ but not for any of the other parameters studied (Table 3 ). Magnitude-based inference adjusted analysis revealed the effect of the PA intervention was likely and very likely to have been beneficial ( $>75 \%$ likelihood of a beneficial effect) on TC and LDL-C, respectively, compared with the usual care (Table 5).

\section{Discussion}

Breast cancer survivors who received a home-based PA intervention significantly increased our primary outcome, self-reported total PA compared with usual care. 
Table 1 Personal characteristics of the participants at baseline (intervention, $n=40$; usual care, $n=40$ )

\begin{tabular}{|c|c|c|c|}
\hline & Participants N (\%) overall & Participants N (\%) intervention & Participants N (\%) usual care \\
\hline Mean $\pm s$ age $(y)$ & $53.6 \pm 9.4$ & $52.4 \pm 10.3$ & $54.7 \pm 8.3$ \\
\hline Mean $\pm s$ time since diagnosis (weeks) & $38.0 \pm 20.8$ & $42.2 \pm 20.0$ & $34.4 \pm 21.1$ \\
\hline Mean $\pm s$ weeks from end of treatment & $10.5 \pm 9.0$ & $8.9 \pm 7.3$ & $12.0 \pm 10.3$ \\
\hline \multicolumn{4}{|l|}{ Ethnic origin: } \\
\hline o White British & $76(95)$ & $38(95)$ & $38(95)$ \\
\hline o White Irish & $1(1)$ & $0(0)$ & $1(3)$ \\
\hline o Other white background & $1(1)$ & $1(3)$ & $0(0)$ \\
\hline o Black Caribbean & $2(3)$ & $1(3)$ & $1(3)$ \\
\hline \multicolumn{4}{|l|}{ BMI $\left(\mathrm{kg} / \mathrm{m}^{2}\right):$} \\
\hline $\mathrm{O} B M \mathrm{I}=\geq 30$ & $22(28)$ & $12(30)$ & $10(25)$ \\
\hline o $\mathrm{BMI}=25-29.9$ & $29(36)$ & $13(33)$ & $16(40)$ \\
\hline $\mathrm{o} B M I=<25$ & $29(36)$ & $15(38)$ & $14(36)$ \\
\hline \multicolumn{4}{|l|}{ Family history of breast cancer: } \\
\hline o Yes & $15(19)$ & $8(20)$ & $7(18)$ \\
\hline o No & $65(81)$ & $32(80)$ & $33(82)$ \\
\hline \multicolumn{4}{|l|}{ Smoking: } \\
\hline o Ever & $33(41)$ & $12(30)$ & $21(52)$ \\
\hline o Never & $47(59)$ & $28(70)$ & $19(48)$ \\
\hline Alcohol drinkers: & $57(71)$ & $28(70)$ & $29(72)$ \\
\hline \multicolumn{4}{|l|}{ Current or previous co-morbidities: } \\
\hline o Diabetes & $3(4)$ & $2(5)$ & $1(3)$ \\
\hline o Hypertension & $12(15)$ & $4(10)$ & $8(20)$ \\
\hline o High cholesterol & $6(8)$ & $3(8)$ & $3(8)$ \\
\hline o Heart disease & $4(5)$ & $2(5)$ & $2(5)$ \\
\hline - Vascular disease & $2(3)$ & $1(3)$ & $1(3)$ \\
\hline o Asthma/chronic bronchitis & $9(11)$ & $3(8)$ & $6(15)$ \\
\hline o Osteoarthritis & $13(16)$ & $4(10)$ & $9(23)$ \\
\hline o Rheumatoid arthritis & $3(4)$ & $2(5)$ & $1(3)$ \\
\hline o Kidney disease & $2(3)$ & $0(0)$ & $2(5)$ \\
\hline Parity & $69(86)$ & $36(45)$ & $33(41)$ \\
\hline Breast fed children & $45(56)$ & $23(58)$ & $22(55)$ \\
\hline Currently menstruating & $16(20)$ & $8(23)$ & $8(20)$ \\
\hline Oral Contraceptive (OC) use (current/previous) & $64(80)$ & $30(75)$ & $34(85)$ \\
\hline Mean $\pm s$ years $O C$ taken & $9.2 \pm 7.8$ & $10.0 \pm 7.9$ & $8.5 \pm 7.7$ \\
\hline Previous/current use of HRT & $21(26)$ & $9(22)$ & $12(30)$ \\
\hline Mean $\pm s$ years taking HRT & $6.9 \pm 4.9$ & $5.9 \pm 3.8$ & $7.6 \pm 5.5$ \\
\hline \multicolumn{4}{|l|}{ Marital status: } \\
\hline o Married/in relationship & $65(81)$ & $36(91)$ & $29(73)$ \\
\hline o Single/divorced/separated/widowed & $15(19)$ & $4(9)$ & $11(27)$ \\
\hline \multicolumn{4}{|l|}{ Highest qualification: } \\
\hline o College degree/diploma and above & $30(40)$ & $15(38)$ & $10(27)$ \\
\hline \multicolumn{4}{|l|}{ Employment status: } \\
\hline o Employed full-time/part-time & $41(53)$ & $21(53)$ & $21(53)$ \\
\hline
\end{tabular}


Table 1 Personal characteristics of the participants at baseline (intervention, $n=40$; usual care, $n=40$ ) (Continued)

\begin{tabular}{llll}
\hline IPAQ physical activity category: & & \\
O Low activity & $15(19)$ & $8(20)$ & $7(18)$ \\
o Moderate activity & $55(69)$ & $30(75)$ & $25(63)$ \\
o High activity & $10(13)$ & $2(5)$ & $8(20)$
\end{tabular}

Key: HRT Hormone Replacement Therapy

Table 2 Effect of physical activity (PA) intervention on PA and anthropometric variables (all PA data reported as MET-min·wk ${ }^{-1}$ )

\begin{tabular}{|c|c|c|c|c|c|c|}
\hline & \multirow[b]{2}{*}{ Baseline mean (SD) } & \multirow[b]{2}{*}{ Follow-up mean (SD) } & \multirow{2}{*}{$\begin{array}{l}\text { Within-group change at follow-up } \\
\text { Mean change }(95 \% \mathrm{Cl})\end{array}$} & \multicolumn{3}{|c|}{ Adjusted between-group change at follow-up } \\
\hline & & & & Mean change (95 \% Cl) & ES & $p$-value \\
\hline \multicolumn{7}{|l|}{ Total PA } \\
\hline Intervention & $1354.74(1073.44)$ & $1899.01(985.84)$ & 530.11 (203.35 to 856.86$)$ & 578.47 (76.09 to 1080.84$)$ & .44 & .024 \\
\hline Usual care & $1974.60(1478.57)$ & $1968.19(1133.87)$ & $-49.66(-532.95$ to 433.62$)$ & Reference & & \\
\hline \multicolumn{7}{|l|}{ Work-based PA } \\
\hline Intervention & $29.12(117.59)$ & $52.54(145.10)$ & $21.06(-20.97$ to 63.09$)$ & $-45.85(-128.47$ to 36.78$)$ & -.10 & .273 \\
\hline Usual care & $203.85(643.40)$ & $241.24(635.99)$ & $66.91(-17.76$ to 151.58$)$ & Reference & & \\
\hline \multicolumn{7}{|c|}{ Active transport PA } \\
\hline Intervention & $167.89(160.67)$ & $222.08(181.90)$ & $42.36(-35.42$ to 120.15$)$ & $23.14(-88.82$ to 135.10$)$ & .10 & .683 \\
\hline Usual care & $223.56(268.35)$ & $255.16(253.96)$ & $19.20(-83.73$ to 122.13$)$ & Reference & & \\
\hline \multicolumn{7}{|l|}{ Domestic PA } \\
\hline Intervention & $623.60(627.00)$ & $729.54(663.90)$ & $93.08(-177.84$ to 364.00$)$ & $153.61(-219.72$ to 526.93$)$ & .23 & .417 \\
\hline Usual care & $850.68(698.20)$ & $793.10(689.33)$ & $-59.33(-393.63$ to 274.97$)$ & Reference & & \\
\hline \multicolumn{7}{|l|}{ Leisure PA } \\
\hline Intervention & $478.76(591.57)$ & $875.42(789.59)$ & $414.03(242.22$ to 585.84$)$ & 382.18 (93.75 to 670.61$)$ & .61 & .010 \\
\hline Usual care & $565.79(671.65)$ & $652.37(529.02)$ & $31.57(-237.19$ to 300.32$)$ & Reference & & \\
\hline \multicolumn{7}{|l|}{ Walking PA } \\
\hline Intervention & $439.64(416.43)$ & 706.56 (789.59) & 289.24 (105.74 to 472.74) & $172.93(-61.75$ to 407.61$)$ & .39 & .147 \\
\hline Usual care & $567.16(472.40)$ & $688.77(528.71)$ & $116.30(-59.49$ to 289.09$)$ & Reference & & \\
\hline \multicolumn{7}{|l|}{ Moderate PA } \\
\hline Intervention & $695.10(673.94)$ & $856.24(675.64)$ & $142.49(-126.52$ to 411.49$)$ & $112.44(-307.39$ to 532.27$)$ & .13 & .597 \\
\hline Usual care & $1087.60(1020.28)$ & $1126.04(911.75)$ & $30.61(-376.54$ to 437.77$)$ & Reference & & \\
\hline \multicolumn{7}{|l|}{ Vigorous PA } \\
\hline Intervention & $142.03(387.19)$ & $275.65(572.14)$ & 122.11 (17.10 to 227.11) & 264.07 (73.51 to 454.64$)$ & .59 & .007 \\
\hline Usual care & $60.60(143.22)$ & $60.61(143.22)$ & $-143.06(-340.84$ to 54.72$)$ & Reference & & \\
\hline \multicolumn{7}{|l|}{ Mass (kg) } \\
\hline Intervention & $70.86(11.83)$ & $69.43(12.46)$ & $-1.61(-2.80$ to -.42$)$ & $-1.62(-3.16$ to -.07$)$ & -.14 & .040 \\
\hline Usual care & $69.15(11.20)$ & $69.19(12.36)$ & $-.00(-1.03$ to 1.04$)$ & Reference & & \\
\hline \multicolumn{7}{|l|}{ BMI $\left(\mathrm{kg} / \mathrm{m}^{2}\right)$} \\
\hline Intervention & $27.25(4.69)$ & $26.58(4.93)$ & $-.62(-1.08$ to -.17$)$ & $-.62(-1.17$ to -.06$)$ & -.14 & .030 \\
\hline Usual care & $26.67(4.04)$ & $26.79(4.2)$ & $-.01(-.41$ to .40$)$ & Reference & & \\
\hline \multicolumn{7}{|l|}{ Body fat \% } \\
\hline Intervention & $35.88(6.46)$ & $36.24(6.61)$ & $.41(-.55$ to 1.37$)$ & $.34(-.93$ to 1.62$)$ & .06 & .594 \\
\hline Usual care & $35.26(5.90)$ & $35.52(6.26)$ & .05 (-.85 to .95$)$ & Reference & & \\
\hline
\end{tabular}

Key: SD indicates standard deviation; $95 \% \mathrm{Cl}, 95 \%$ confidence interval; ES, effect size (Cohen's d; effect estimate/pooled baseline SD) Baseline means (SD) are based on 80 participants (intervention $=40$; usual care $=40$ ); post-interventions and within-group change at follow-up means (SD) are based on 70 participants (intervention $=37$; control $=33$ )

Except for the anthropometric measures, positive ES indicate effects in favour of the exercise intervention group

Between-group effects were assessed using linear mixed model analysis, adjusted for the value of the outcome variable at baseline 
Table 3 Effect of PA intervention on HRQOL FACT-B and blood biomarker variables

\begin{tabular}{|c|c|c|c|c|c|c|}
\hline & \multirow[b]{2}{*}{ Baseline mean (SD) } & \multirow[b]{2}{*}{ Follow-up mean (SD) } & \multirow{2}{*}{$\begin{array}{l}\text { Within-group change at } \\
\text { follow-up } \\
\text { Mean change ( } 95 \% \text { Cl) }\end{array}$} & \multicolumn{3}{|c|}{$\begin{array}{l}\text { Adjusted between-group change } \\
\text { at follow-up }\end{array}$} \\
\hline & & & & Mean change (95 \% Cl) & ES & $p$-value \\
\hline \multicolumn{7}{|l|}{ FACT-B } \\
\hline Intervention & $108.56(21.97)$ & $114.41(21.48)$ & 5.91 (1.88 to 9.93$)$ & 5.05 (.69 to 9.40$)$ & .25 & .024 \\
\hline Usual care & $114.25(18.97)$ & $115.34(17.57)$ & $.56(-3.43$ to 4.56$)$ & Reference & & \\
\hline \multicolumn{7}{|l|}{ FACT-G } \\
\hline Intervention & $85.75(17.35)$ & $88.03(18.53)$ & $2.62(-2.72$ to 7.96$)$ & $2.17(-1.43$ to 5.78$)$ & .14 & .234 \\
\hline Usual care & $89.30(13.23)$ & $89.59(16.84)$ & $-.09(-5.65$ to 5.46$)$ & Reference & & \\
\hline \multicolumn{7}{|l|}{ TOI } \\
\hline Intervention & $65.29(15.09)$ & $72.59(15.13)$ & 7.20 (4.47 to 9.93$)$ & 5.64 (2.33 to 8.95$)$ & .39 & .001 \\
\hline Usual care & $71.13(13.07)$ & $73.25(16.52)$ & $2.13(-1.89$ to 6.14$)$ & Reference & & \\
\hline \multicolumn{7}{|l|}{ PWB } \\
\hline Intervention & $22.03(5.25)$ & $25.54(9.32)$ & $3.43(.58$ to 6.29$)$ & $.63(-.87$ to 2.13$)$ & .13 & .404 \\
\hline Usual care & $23.03(4.39)$ & $25.84(9.18)$ & $2.84(-.15$ to 5.84$)$ & Reference & & \\
\hline \multicolumn{7}{|l|}{ SWB } \\
\hline Intervention & $24.35(4.44)$ & $23.70(5.03)$ & $-.54(-1.40$ to .32$)$ & $-.73(-2.03$ to .59$)$ & .17 & .276 \\
\hline Usual care & $23.40(4.19)$ & $24.13(4.13)$ & $.19(-1.00$ to 1.37$)$ & Reference & & \\
\hline \multicolumn{7}{|l|}{ EWB } \\
\hline Intervention & $18.93(4.61)$ & $20.11(4.14)$ & 1.24 (.46 to 2.03$)$ & $.20(-.85$ to 1.26$)$ & .05 & .701 \\
\hline Usual care & $19.73(3.74)$ & $20.72(3.30)$ & $1.00(.20$ to 1.80$)$ & Reference & & \\
\hline \multicolumn{7}{|l|}{ FWB } \\
\hline Intervention & $20.45(6.10)$ & $21.95(4.41)$ & $1.76(.21$ to 3.30$)$ & $1.90(.24$ to 3.55$)$ & .36 & .025 \\
\hline Usual care & $23.15(3.94)$ & $22.88(4.70)$ & $-.16(-1.25$ to .94$)$ & Reference & & \\
\hline \multicolumn{7}{|l|}{ BCS } \\
\hline Intervention & $22.81(6.67)$ & $25.24(7.13)$ & 2.15 (.98 to 3.32$)$ & 2.84 (.79 to 4.89$)$ & .42 & .007 \\
\hline Usual care & $24.95(6.79)$ & $24.34(6.21)$ & $-75(-2.81$ to 1.31$)$ & Reference & & \\
\hline \multicolumn{7}{|l|}{$\mathrm{TC}\left(\mathrm{mmol} \cdot \mathrm{L}^{-1}\right)$} \\
\hline Intervention & $5.75(1.30)$ & $5.4(1.09)$ & $-.23(-.49$ to .04$)$ & $-.45(-.71$ to -.18$)$ & -.38 & .001 \\
\hline Usual care & $5.79(1.04)$ & $5.93(.91)$ & $.22(.05$ to .38$)$ & Reference & & \\
\hline \multicolumn{7}{|l|}{$\mathrm{HDL}\left(\mathrm{mmol} \cdot \mathrm{L}^{-1}\right)$} \\
\hline Intervention & $1.65(.30)$ & $1.62(.33)$ & $-.02(-.12$ to 0.79$)$ & $-.06(-.15$ to .04$)$ & -.19 & .264 \\
\hline Usual care & $1.59(.34)$ & $1.60(.30)$ & $.04(-.03$ to .10$)$ & Reference & & \\
\hline \multicolumn{7}{|l|}{$\mathrm{LDL}\left(\mathrm{mmo} \cdot \mathrm{L}^{-1}\right)$} \\
\hline Intervention & $3.44(1.15)$ & $3.18(1.01)$ & $-.19(-.42$ to .04$)$ & $-.30(-.56$ to -.04$)$ & -.30 & .023 \\
\hline Usual care & $3.56(.84)$ & $3.64(.84)$ & $.11(-.11$ to .32$)$ & Reference & & \\
\hline \multicolumn{7}{|c|}{$\mathrm{TC} / \mathrm{HDL}-\mathrm{C}$ ratio $\left(\mathrm{mmol} \cdot \mathrm{L}^{-1}\right)$} \\
\hline Intervention & $3.61(1.12)$ & $3.47(.93)$ & $-.09(-.36$ to .09$)$ & $-.13(-.40$ to .14$)$ & -.12 & .337 \\
\hline Usual care & $3.79(1.00)$ & $3.83(.90)$ & $.04(-.19$ to .26$)$ & Reference & & \\
\hline \multicolumn{7}{|l|}{ Trig $\left(\mathrm{mmol} \cdot \mathrm{L}^{-1}\right)$} \\
\hline Intervention & $1.43(.75)$ & $1.31(.58)$ & $-.08(-.21$ to .05$)$ & $-.10(-.27$ to .07$)$ & -.13 & .240 \\
\hline Usual care & $1.52(.85)$ & $1.52(.73)$ & $.02(-.14$ to .18$)$ & Reference & & \\
\hline \multicolumn{7}{|c|}{ Glucose $\left(\mathrm{mmol} \cdot \mathrm{L}^{-1}\right)$} \\
\hline Intervention & $4.94(.85)$ & $4.81(.52)$ & $-.14(-.36$ to .09$)$ & $-.07(-.38$ to .25$)$ & -.05 & .683 \\
\hline Usual care & $5.27(1.96)$ & $5.32(2.20)$ & $-.08(-.40$ to .25$)$ & Reference & & \\
\hline
\end{tabular}


Table 3 Effect of PA intervention on HRQOL FACT-B and blood biomarker variables (Continued)

\begin{tabular}{|c|c|c|c|c|c|c|}
\hline \multicolumn{7}{|c|}{ Insulin $\left(\mathrm{pmol} \cdot \mathrm{L}^{-1}\right)$} \\
\hline Intervention & $38.37(34.61)$ & $37.40(28.64)$ & -1.03 (15.37 to 13.31$)$ & 8.31 (-12.31 to 28.95$)$ & \multirow[t]{2}{*}{.20} & \multirow[t]{2}{*}{.425} \\
\hline Usual care & 45.45 (48.69) & $37.34(40.60)$ & $-9.84(-29.76$ to 10.08$)$ & Reference & & \\
\hline \multicolumn{7}{|l|}{ HOMA } \\
\hline Intervention & $1.60(1.18)$ & $1.78(.88)$ & $.07(-.62$ to .76$)$ & $.42(-.88$ to 1.73$)$ & \multirow[t]{2}{*}{.27} & \multirow[t]{2}{*}{.520} \\
\hline Usual care & $2.18(1.83)$ & $1.90(2.09)$ & $-.41(-2.09$ to 1.26$)$ & Reference & & \\
\hline \multicolumn{7}{|c|}{$\begin{array}{l}\text { Key: FACT-G = PWB + SWB + EWB + FWB; FACT-B = FACT-G + BCS; TOI = PWB + FWB + BCS } \\
\text { Baseline means (SD) are based on } 80 \text { participants except for HOMA }(N=52 ; \text { intervention }=27 ; \text { control }=25) \text {; Post-interventions and with-group change at follow-ur } \\
\text { means (SD) are based on } 70 \text { participants (intervention = 37; control = 33), except HOMA }(N=38 ; \text { Intervention }=20 ; \text { control }=18) \\
\text { Except for the blood measures (Higher scores represent better quality of life), positive ES indicate effects in favour of the exercise intervention group } \\
\text { Between-group effects were assessed using linear mixed model analysis including the measurements obtained at baseline and post-intervention, adjusted for the } \\
\text { value of the outcome variable at baseline }\end{array}$} \\
\hline
\end{tabular}

We also found further significant improvements in leisure and vigorous PA, body mass, BMI, HRQoL (FACT-B, TOI, FWB, and BCS) and TC and LDL-C concentrations in the intervention compared with usual care. All of the significant improvements above were found to have small effect sizes, apart from the moderate effects observed for leisure and vigorous PA. However, the difference in improvement in total PA for the intervention group (578 MET-min. $\mathrm{wk}^{-1}$ ) compared to the usual care group was close to the recommended PA guidelines of $600 \mathrm{MET}-\mathrm{min} \cdot \mathrm{wk}^{-1}$, i.e. $5 \times 30 \mathrm{~min}$ of moderate PA). Therefore, this improvement would result in more breast cancer survivors meeting recommended PA guidelines, and possibly deriving associated benefits of reduced risk of mortality and recurrence [9-12]. Of note, more participants in the intervention group experienced minimum clinically important improvements in TOI and BCS compared with the usual care group. However, we observed no significant improvements in any other PA variables, body fat, and other HRQoL and blood biomarker variables.

Our findings of increases in total, leisure-based, and vigorous $\mathrm{PA}$ are consistent with previous US homebased PA interventions with an additional PA counselling element $[17,20,22]$. All of these trials were relatively short in duration (12 weeks) compared with the duration of the current study (6 months). The increases in PA found in the current study were encouraging given the larger sample size of invasive breast cancer survivors. However, unlike two previous studies $[20,27]$, we found no significant differences in selfreported walking from baseline to post-intervention in the intervention group compared with the usual care group. This was possibly due to contamination in the usual care group since these individuals were made aware of recommended PA guidelines at baseline, as it was thought unethical to withhold this information considering the potential health benefits associated with PA.

Table 4 Changes in performance and anthropometric measures in experimental and control groups and qualitative inferences about the intervention effects

\begin{tabular}{|c|c|c|c|c|c|}
\hline \multirow[t]{2}{*}{ Variable } & \multicolumn{4}{|c|}{ Change in measure (\%) from baseline to post-intervention } & \multirow[b]{2}{*}{$\begin{array}{l}\text { Qualitative inference (\% likelihood } \\
\text { of at least a small effect) }\end{array}$} \\
\hline & $\begin{array}{l}\text { Intervention } \\
\text { mean (SD) }\end{array}$ & $\begin{array}{l}\text { Usual care mean } \\
\text { (SD as a CV) }\end{array}$ & $\begin{array}{l}\text { Between group } \\
\text { difference }(90 \% \mathrm{Cl})\end{array}$ & Effect size $(d)$ & \\
\hline Total PA & $55.9(209.5)$ & $17.3(93.0)$ & $32.9(-9.0$ to 94.0$)$ & $.31(-.10$ to .72$)$ & Possibly beneficial (67) \\
\hline Work-based PA & $-6.3(814.8)$ & $51.2(327.1)$ & $-56.2(-84.0$ to 19.8$)$ & $-.33(-.73$ to .07$)$ & Possibly decreases (52) \\
\hline Active transport PA & $-24.6(783.9)$ & $72.3(1452.7)$ & $-44.7(-79.9$ to 52.2$)$ & $-.23(-.63$ to .16$)$ & Possibly decreases (70) \\
\hline Domestic PA & $81.3(652.3)$ & $-11.4(768.2)$ & $104.6(-12.5$ to 378.7$)$ & $.37(-.07$ to .81$)$ & Possibly beneficial (74) \\
\hline Leisure PA & $408.0(1208.6)$ & $208.9(1615.0)$ & $64.8(-44.0$ to 384.9$)$ & $.19(-.23$ to .61$)$ & Possibly beneficial (50) \\
\hline Walking PA & $76.2(851.2)$ & $70.7(916.4)$ & $3.3(-59.0$ to 160.1$)$ & $.02(-.47$ to .50$)$ & Possibly beneficial (51) \\
\hline Moderate PA & 71.1 (477.9) & $-18.2(527.5)$ & 109.1 (-.2 to 338.2) & $.41(.00$ to .83$)$ & Likely beneficial (80) \\
\hline Vigorous PA & 137.6 (2078.0) & $33.6(868.1)$ & 77.8 (-39.2 to 420.0) & $.22(-0.19$ to .62$)$ & Possibly beneficial (53) \\
\hline Mass & $-2.3(4.7)$ & $-.1(4.1)$ & $-2.2(-3.9$ to -.5$)$ & $-.13(-.24$ to -.03$)$ & Unlikely beneficial (14) \\
\hline BMI & $-2.3(4.6)$ & $-.1(4.1)$ & $-2.2(-3.9$ to -.5$)$ & $-.14(-.25$ to -.03$)$ & Unlikely beneficial (18) \\
\hline Body fat \% & $1.3(8.0)$ & $-.1(7.5)$ & $1.4(-1.6$ to 4.4$)$ & $.08(-.09$ to .25$)$ & Unlikely increases (11) \\
\hline
\end{tabular}


Table 5 Changes in HRQOL and blood biomarkers measures in experimental and control groups and qualitative inferences about the intervention effects

\begin{tabular}{|c|c|c|c|c|c|}
\hline \multirow[t]{2}{*}{ Variable } & \multicolumn{4}{|c|}{ Change in measure (\%) from baseline to post-intervention } & \multirow[b]{2}{*}{$\begin{array}{l}\text { Qualitative inference (\% likelihooc } \\
\text { of at least a small effect) }\end{array}$} \\
\hline & $\begin{array}{l}\text { Intervention } \\
\text { mean (SD) }\end{array}$ & $\begin{array}{l}\text { Usual care mean } \\
\text { (SD as a CV) }\end{array}$ & $\begin{array}{l}\text { Between group } \\
\text { difference }(90 \% \mathrm{Cl})\end{array}$ & Effect size $(d)$ & \\
\hline Fact-B & $5.9(10.8)$ & $2.1(7.9)$ & $3.7(.0$ to 7.5$)$ & $.18(.00$ to .35$)$ & Possibly beneficial (59) \\
\hline Fact-G & $16.2(85.1)$ & $2.7(8.8)$ & $13.1(-4.9$ to 34.6$)$ & $.34(-.14$ to .82$)$ & Possibly beneficial (69) \\
\hline TOI & $4.8(31.1)$ & $1.6(10.2)$ & $3.1(-4.8$ to 11.8$)$ & $.13(-.21$ to .47$)$ & Possibly beneficial (36) \\
\hline BCS & $8.8(18.5)$ & $1.0(28.1)$ & $7.7(-1.2$ to 17.5$)$ & $.22(-.04$ to .47$)$ & Possibly beneficial (54) \\
\hline PWB & $9.2(16.2)$ & $7.6(14.6)$ & $1.5(-4.2$ to 7.5$)$ & $.07(-.19$ to .33$)$ & Unlikely beneficial (20) \\
\hline SWB & $-2.5(12.3)$ & $.7(13.3)$ & $-3.2(-7.8$ to 1.6$)$ & $-.15(-.37$ to .07$)$ & Possibly decreases (36) \\
\hline EWB & $5.6(13.5)$ & $5.6(9.7)$ & $-.0(-4.3$ to 4.5$)$ & $.00(-0.19$ to .19$)$ & Very unlikely beneficial (5) \\
\hline FWB & $6.2(20.4)$ & $-1.3(15.0)$ & 7.6 (.6 to 15.2$)$ & $.26(.02$ to .50$)$ & Possibly beneficial (66) \\
\hline TC & $-3.5(10.5)$ & $3.2(6.7)$ & $-6.5(-9.6$ to -3.3$)$ & $-.41(-.62$ to -.21$)$ & Very likely beneficial (95) \\
\hline $\mathrm{HDL}-\mathrm{C}$ & $-.7(11.0)$ & $1.0(6.7)$ & $-1.7(-5.0$ to 1.9$)$ & $-.14(-.42$ to .15$)$ & Possibly beneficial (35) \\
\hline LDL-C & $-4.5(14.9)$ & $2.5(12.8)$ & $-6.8(-11.6$ to -1.8$)$ & $-.31(-.55$ to -.08$)$ & Likely beneficial (79) \\
\hline TC/LDL-C ratio & $-2.3(14.5)$ & $1.6(11.3)$ & $-3.8(-8.5$ to 1.0$)$ & $-.19(-.43$ to .05$)$ & Possibly beneficial (47) \\
\hline Trig & $-3.5(16.3)$ & $1.8(16.4)$ & $-5.2(-10.9$ to .8$)$ & $-.20(-.42$ to .03$)$ & Possibly beneficial (49) \\
\hline Glucose & $-2.9(6.7)$ & $-6.1(40.6)$ & $3.4(-6.7$ to 14.5$)$ & $.14(-.30$ to .59$)$ & Possibly beneficial (42) \\
\hline Insulin & $-3.6(87.3)$ & $-24.2(145.4)$ & $27.2(-7.1$ to 74.3$)$ & $.31(-.09$ to .70$)$ & Possibly increases (67) \\
\hline HOMA & $-4.5(39.5)$ & $0.7(61.6)$ & $-5.2(-26.6$ to 22.5$)$ & $-.12(-.72$ to .47$)$ & Possibly beneficial (41) \\
\hline
\end{tabular}

Key: $C V=$ coefficient of variation

Beneficial effect reflects an increase in HRQoL measures and a decrease in blood biomarkers

Therefore, being part of the current study may have increased the awareness of health benefits associated with $\mathrm{PA}$ in the usual care group and resulted in increased walking activity. Another possible reason for this is that the IPAQ assesses walking in occupational and active transport domains as well as leisure domains, in which usual care participants engaged in more than the intervention participants. Similarly, a greater amount of occupational and active transport may also explain the non-significant between-group differences in moderate PA, although the per-protocol analysis revealed a likely beneficial effect of the intervention on moderate PA. It is also important to note that, on average, the PA levels of the intervention group were lower than that of the usual care group at baseline, and therefore, it can be argued that they had a greater room for improvement. However, the higher self-reported PA levels of the usual care group was largely the result of three participants in this group who reported much higher PA levels $(\geq 5000$ MET-min $\cdot \mathrm{wk}^{-1}$ ) compared to other participants. Therefore, with the exception of these three participants the levels of PA were similar in both groups, as evidenced by the number of participants in the low and moderate PA categories in each group (see Table 1).

Unlike similar previous studies, we found significant reductions in body mass and BMI [17, 20, 22]. Although the effects of the intervention compared with usual care could be considered trivial for both outcomes, it does represent at the least, more effective weight management. The significant albeit small reductions in mass and BMI were surprising given that the intervention did not focus on weight loss and did not involve any calorie restriction. However, previous research has found positive associations between PA and healthy eating behaviours [44], which in turn may have had a beneficial effect on body mass and BMI. In addition, this finding could be due in part to the longer duration of the current study compared with the earlier studies (6 months vs. 12 weeks) $[17,20,22,27]$. However, we found no significant improvements in body fat \% despite the improvements in mass and BMI. It is possible that the method of assessing body fat \%, bioelectrical impedance is not a precise enough method to measure small changes in body fat over time [45].

We observed significant small increases in the HRQoL variables, FACT-B, TOI, FWB and BCS in the intervention group compared to the usual care group. In addition, a greater proportion of participants in the intervention group achieving minimum clinical significant improvements in BSC and TOI, which indicates that our intervention may have specific benefits for breast cancer survivors given that higher BCS scores indicate fewer breast cancer-specific symptoms, such as 'shortness of breath', 'change in weight' and 'effect of stress on illness', and higher TOI scores indicate both greater physical and functional well-being and fewer 
breast cancer-specific symptoms. A methodologically similar study reported significantly greater improvements for social well-being in the intervention group versus usual care group, but no significant improvements were found in the other HRQoL variables [17]. Significant improvements in the FACT-B variable in the intervention group versus usual care group was reported by an earlier home-based PA trial involving breast cancer survivors, which did not have a counselling component [26]. The reasons for the differences between previous studies are unclear. However, it is likely that the breast cancer stage, treatment received and both the time since diagnosis and the end of treatment may influence participant's responses to the items with the FACT questionnaire.

Our findings of significant but small reductions in TC and LDL-C were encouraging given that the existing literature investigating the prevention of cardiovascular disease (CVD) emphasizes the role of TC and LDL-C with a supplementary role for HDL-C and a modest role for TG [46-48]. Evidence supports our finding that increased PA can favourably influence lipid profiles [49-51]. The effect of aerobic exercise on glucoseinsulin dynamics is unclear due to a lack of agreement between available studies [52-55]. We may not have found reductions in glucose, insulin, and IR because of an insufficient sample size to detect differences between groups as the trial was not powered to detect changes in these outcomes, an insufficient reduction in body fat [55], and/or the fact that the majority of participants did not have diabetes [56].

The strengths of the current study include its randomised design, the independent randomisation, a pragmatic intervention and the intention-to-treat approach. We also recruited participants over two full calendar years; therefore, it is unlikely that our results were influenced by the seasonal changes in PA suspected in previous research [26]. The limitations of this study include lack of controlling for increased risk of type I errors when making multiple comparisons. Moreover, selfreport measures, such as IPAQ, require participants to recall past activity, are a subjective means of estimating individual PA levels and are reliant on the individuals' ability to remember levels of exposure [57]. Outcomes were assessed by a fully trained exercise scientist, who followed an objective, standardized assessment protocol. However, the scientist was not blinded to group allocation which may have introduced measurement bias. In addition, there was evidence of some contamination in the usual care group. Six of the usual care group increased their PA levels enough to move to a higher PA category from baseline to post-intervention. This contamination may have been because we informed participants in the usual care of the current recommended PA guidelines and we did not discourage them from engaging in PA. We did not control for possible changes in the dietary habits of participants, and it is possible that participants in the intervention group may have also changed to healthier eating behaviours when becoming more physically activity.

\section{Conclusions}

Within the context of these limitations, we found that a home-based PA intervention resulted in significant but small to moderate favourable effects on self-reported PA, body mass, BMI, HRQoL, TC and LDL-C. The results of the trial were promising given that the intervention was relatively brief, pragmatic and highly feasible given that it was home-based and consisted of a single in-person counselling session followed by three support telephone calls. The portability and feasibility of the current intervention means it could be implemented within the NHS framework of breast cancer treatment and follow-up both in the primary and secondary care settings. This has the potential to capture the maximum number of breast cancer survivors can benefit with minimal burden to staff.

\section{Availability of data and materials}

The datasets supporting the conclusions of this article are available in the Figshare repository (https://figshare.com/articles/Randomised_controlled_trial_of_a_home_based_physical_activity_intervention_in_breast_cancer_survivors/308 2987).

\section{Abbreviations}

BCS: Breast cancer subscale score; BMI: Body mass index; EWB: Emotional well-being; FACT-B: FACT-breast; FACT-G: Functional assessment of cancer therapy - general; FWB: Functional well-being; HDL-C: High-density

lipoprotein; HOMA: Homeostasis Model Assessment; HRQoL: Health-related quality of life; HRT: Hormone Replacement Therapy; IR: Insulin resistance; LDL-C: Low-density lipoprotein cholesterol; PA: Physical activity;

PWB: Physical well-being; RCT: Randomised controlled trials; SWB: Social well-being; TC: Total cholesterol; TG: Triglycerides; TOI: Trial outcome index.

\section{Competing interests}

The authors declare that they have no competing interests.

\section{Authors' contributions}

The contributions of each author to the manuscript are: ARC, IML, GSM and GDK conceived and designed the study. IML, GSM and ARC were involved in the data acquisition. All authors (IML, GSM, AMN, GDK, ARC) contributed to data analysis and interpretation. All authors (IML, GSM, AMN, GDK, ARC) contributed to the writing of the report and read and approved the final manuscript.

\section{Acknowledgement}

We would like to express gratitude to Dr Sarah Bowden and her team at the Birmingham Clinical Trials Unit for their support in randomisation, Russells Hall Hospital Breast Care Nurses for helping with recruitment, Russells Hall Hospital phlebotomy department and biochemistry laboratory for assistance with blood collection and analysis, and above all, each of the patients who contributed with their time and feedback to make this study possible. This trial was funded internally by the Department of Research and Development, Dudley Group NHS Foundation Trust, and received no external funding. 


\section{Author details}

${ }^{1}$ Faculty of Education, Health and Wellbeing, University of Wolverhampton, Walsall Campus, Gorway Road, Walsall WS1 3BD, UK. 'Department of Surgery, Russells Hall Hospital, Dudley DY1 2HQ, UK. ${ }^{3}$ Department of Research and Development, Dudley Group NHS Foundation Trust, Russells Hall Hospital, Dudley DY1 2HQWest Midlands, UK.

\section{Received: 16 February 2015 Accepted: 8 March 2016}

\section{Published online: 17 March 2016}

\section{References}

1. Jemal A, Bray F, Center MM, Ferlay J, Ward E, Forman D. Global cancer statistics. CA. 2011:61(2):69-90. doi:10.3322/caac.20107.

2. Office for National Statistics. Cancer incidence and mortality 2007-09. In: Office for National Statistics Statistical Bulletin. Office for National Statistics. 2012. http://www.ons.gov.uk/ons/dcp171778_259504.pdf. Accessed 17 Feb 2015.

3. Moller H, Fairley L, Coupland V, Okello C, Green M, Forman D, et al. The future burden of cancer in England: incidence and numbers of new patients in 2020. Br J Cancer. 2007:96(9):1484-8. doi:10.1038/sj.bjc.6603746.

4. Office for National Statistics. Cancer survival by cancer network in england patients diagnosed 1996-2009 and followed up to 2010. In: Office for National Statistics Statistical Bulletin. Office for National Statistics. 2011. http://www.ons.gov.uk/ons/dcp171778_247385.pdf. Accessed 17 Feb 2015.

5. Azim Jr HA, de Azambuja E, Colozza M, Bines J, Piccart MJ. Long-term toxic effects of adjuvant chemotherapy in breast cancer. Ann Oncol. 2011;22(9): 1939-47. doi:10.1093/annonc/mdq683.

6. Bovelli D, Plataniotis G, Roila F, Group EGW. Cardiotoxicity of chemotherapeutic agents and radiotherapy-related heart disease: ESMO Clinical Practice Guidelines. Ann Oncol. 2010;21 Suppl 5:v277-82. doi:10.1093/annonc/mdq200.

7. van Dalen EC, Michiels EM, Caron HN, Kremer LC. Different anthracycline derivates for reducing cardiotoxicity in cancer patients. Cochrane Database Syst Rev. 2010;5:CD005006. doi:10.1002/14651858.CD005006.pub4.

8. Demark-Wahnefried W, Morey MC, Clipp EC, Pieper CF, Snyder DC, Sloane R, et al. Leading the Way in Exercise and Diet (Project LEAD): intervening to improve function among older breast and prostate cancer survivors. Control Clin Trials. 2003;24(2):206-23.

9. Lahart I, Metsios G, Nevill A, Carmichael A. Physical activity, risk of death and recurrence in breast cancer survivors: A systematic review and meta-analysis of epidemiological studies. Acta Oncol. 2015;5(54):635-54. doi:10.3109/ 0284186X.2014.998275

10. Ballard-Barbash R, Hunsberger S, Alciati MH, Blair SN, Goodwin PJ, McTiernan A, et al. Physical activity, weight control, and breast cancer risk and survival: clinical trial rationale and design considerations. J Natl Cancer Inst. 2009;101(9): 630-43. doi:10.1093/jnci/djp068.

11. Ibrahim EM, Al-Homaidh A. Physical activity and survival after breast cancer diagnosis: meta-analysis of published studies. Med Oncol. 2011;28(3):753-65. doi:10.1007/s12032-010-9536-x.

12. Beasley JM, Newcomb PA, Trentham-Dietz A, Hampton JM, Bersch AJ, Passarelli MN, et al. Post-diagnosis dietary factors and survival after invasive breast cancer. Breast Cancer Res Treat. 2011;128(1):229-36. doi:10.1007/ s10549-010-1323-z

13. Lahart IM, Metsios GS, Nevill AM, Carmichael AR. Physical activity levels in women attending breast screening, receiving chemotherapy and postbreast cancer treatment; a cross-sectional study. Int J Environ Res Public Health. 2014:11(5):5487-96. doi:10.3390/ijerph110505487.

14. Irwin ML, Crumley D, McTiernan A, Bernstein L, Baumgartner R, Gilliland FD, et al. Physical activity levels before and after a diagnosis of breast carcinoma: the Health, Eating, Activity, and Lifestyle (HEAL) study. Cancer. 2003:97(7):1746-57. doi:10.1002/cncr.11227.

15. Irwin ML, McTiernan A, Bernstein L, Gilliland FD, Baumgartner R, Baumgartner K, et al. Physical activity levels among breast cancer survivors. Med Sci Sports Exerc. 2004;36(9):1484-91.

16. Cadmus LA, Salovey P, Yu H, Chung G, KasI S, Irwin ML. Exercise and quality of life during and after treatment for breast cancer: results of two randomized controlled trials. Psycho-Oncology. 2009;18(4):343-52. doi:10.1002/pon.1525.

17. Rogers LQ, Hopkins-Price P, Vicari S, Pamenter R, Courneya KS, Markwell S, et al. A randomized trial to increase physical activity in breast cancer survivors. Med Sci Sports Exerc. 2009:41(4):935-46. doi:10.1249/MSS. Ob013e31818e0e1b.

18. Payne JK, Held J, Thorpe J, Shaw H. Effect of exercise on biomarkers, fatique, sleep disturbances, and depressive symptoms in older women with breast cancer receiving hormonal therapy. Oncol Nurs Forum. 2008;35(4):635-42. doi:10.1188/08.ONF.635-642.

19. Daley AJ, Crank H, Saxton JM, Mutrie N, Coleman R, Roalfe A. Randomized trial of exercise therapy in women treated for breast cancer. J Clin Oncol. 2007;25(13):1713-21. doi:10.1200/JCO.2006.09.5083.

20. Matthews CE, Wilcox S, Hanby CL, Der Ananian C, Heiney SP, Gebretsadik T, et al. Evaluation of a 12-week home-based walking intervention for breast cancer survivors. Support Care Cancer. 2007;15(2):203-11. doi:10.1007/ s00520-006-0122-x.

21. Herrero F, San Juan AF, Fleck SJ, Balmer J, Perez M, Canete S, et al. Combined aerobic and resistance training in breast cancer survivors: a randomized, controlled pilot trial. Int J Sports Med. 2006;27(7):573-80. doi:10.1055/ s-2005-865848.

22. Pinto BM, Frierson GM, Rabin C, Trunzo JJ, Marcus BH. Home-based physical activity intervention for breast cancer patients. J Clin Oncol. 2005;23(15): 3577-87. doi:10.1200/JCO.2005.03.080.

23. Courneya KS, Mackey JR, Bell GJ, Jones LW, Field CJ, Fairey AS. Randomized controlled trial of exercise training in postmenopausal breast cancer survivors: cardiopulmonary and quality of life outcomes. J Clin Oncol. 2003; 21(9):1660-8. doi:10.1200/JCO.2003.04.093.

24. Musanti R. A study of exercise modality and physical self-esteem in breast cancer survivors. Med Sci Sports Exerc. 2012;44(2):352-61. doi:10.1249/MSS Ob013e31822cb5f2

25. Heim ME, $\vee$ d Malsburg ML, Niklas A. Randomized controlled trial of a structured training program in breast cancer patients with tumorrelated chronic fatigue. Onkologie. 2007;30(8-9):429-34. doi:10.1159/ 0000104097.

26. Vallance JK, Courneya KS, Plotnikoff RC, Yasui Y, Mackey JR. Randomized controlled trial of the effects of print materials and step pedometers on physical activity and quality of life in breast cancer survivors. J Clin Oncol. 2007;25(17):2352-9. doi:10.1200/JCO.2006.07.9988.

27. Baruth M, Wilcox S, Der Ananian C, Heiney S. Effects of home-based walking on quality of life and fatigue outcomes in early stage breast cancer survivors: A 12-Week pilot study. J Phys Act Health. 2013;12 Suppl 1:S110-8. doi:10.1123/ jpah.2012-0339.

28. Glasgow R, Bull S, Gillette C, Klesges L, Dzewaltowski D. Behavior change intervention research in healthcare settings: a review of recent reports with emphasis on external validity. Am J Prev Med. 2002;23(1):62-9. doi:10.1016/ S0749-3797(02)00437-3.

29. Patsopoulos NA. A pragmatic view on pragmatic trials. Dialogues Clin Neurosci. 2011:13(2):217-24

30. Medicine ACOS. ACSM's Guidelines for exercise testing and prescription. 9th ed. Philadelphia, PA: Wolters Kluwer/Lippincott Williams \& Wilkins; 2013.

31. Rogers LQ, Markwell S, Hopkins-Price P, Vicari S, Courneya KS, Hoelzer K, et al. Reduced barriers mediated physical activity maintenance among breast cancer survivors. J Sport Exerc Psychol. 2011;33(2):235-54.

32. Jones LW, Courneya KS. Exercise counseling and programming preferences of cancer survivors. Cancer Pract. 2002;10(4):208-15.

33. Rosengren DB. Building motivational interviewing skills: a practitioner workbook. New York; London: Guilford; 2009.

34. Miller WR, Rollnick S. Motivational interviewing: helping people change. 3rd ed. New York, NY: Guilford Press; 2013.

35. Bull FC, Experts Working Groups. Physical Activity Guidelines in the UK. Review and Recommendations. School of Sport, Exercise and Health Sciences, Loughborough University. 2010. https://www.gov.uk/government/ uploads/system/uploads/attachment_data/file/213743/dh_128255.pdf. Accessed 17 Feb 2015

36. Demura S, Yamaji S, Goshi F, Kobayashi H, Sato S, Nagasawa Y. (2002) The validity and reliability of relative body fat estimates and the construction of new prediction equations for young Japanese adult males. J Sports Sci. 2002;20(2):153-64. doi:10.1080/026404102317200864

37. Craig C, Marshall A, Sjöström M, Bauman A, Booth M, Ainsworth B, et al. International physical activity questionnaire: 12-country reliability and validity. Med Sci Sports Exerc. 2003:8(35):1381-95.

38. Brady MJ, Cella DF, Mo F, Bonomi AE, Tulsky DS, Lloyd SR, et al. Reliability and validity of the Functional Assessment of Cancer Therapy-Breast (FACT-B) quality of life instrument. J Clin Oncol. 1997;15:974-86.

39. Radikova Z. Assessment of insulin sensitivity/resistance in epidemiological studies. Endocr Regul. 2003;37(3):189-94.

40. Batterham A, Hopkins W. Making meaningful inferences about magnitudes. Int J Sports Physiol Perform. 2006;1(1):50-7. 
41. Hopkins WG. Spreadsheets for analysis of controlled trials, with adjustment for a subject characteristic. Sportsci. 2006;10:46-50.

42. Eton DT, Cella D, Yost K, Yount SE, Peterman AH, Neuberg DS, et al. A combination of distribution- and anchor-based approaches determined minimally important differences (MIDs) for four endpoints in a breast cancer scale. J Clin Epidemiol. 2004;57(9):898-910. doi:10.1016/j.jclinepi.2004.01.012.

43. Cohen J. A power primer. Psychol Bull. 1992;1(112):155-9.

44. Pate RR, Heath GW, Dowda M, Trost SG. Associations between physical activity and other health behaviors in a representative sample of US adolescents. Am J Public Health. 1996;86(11):1577-81.

45. Dehghan M, Merchant AT. Is bioelectrical impedance accurate for use in large epidemiological studies? Nutr J. 2008;7:26. doi:10.1186/1475-2891-7-26.

46. Catapano AL, Reiner Z, De Backer G, Graham I, Taskinen MR, Wiklund O, et al. ESC/EAS Guidelines for the management of dyslipidaemias The Task Force for the management of dyslipidaemias of the European Society of Cardiology (ESC) and the European Atherosclerosis Society (EAS). Atherosclerosis. 2011:217(1):3-46.

47. O'Keefe Jr JH, Cordain L, Harris WH, Moe RM, Vogel R. Optimal low-density lipoprotein is 50 to $70 \mathrm{mg} / \mathrm{dl}$ : lower is better and physiologically normal. J Am Coll Cardiol. 2004;43(11):2142-6. doi:10.1016/j.jacc.2004.03.046.

48. Edwards JE, Moore RA. Statins in hypercholesterolaemia: a dose-specific meta-analysis of lipid changes in randomised, double blind trials. BMC Fam Pract. 2003:4:18. doi:10.1186/1471-2296-4-18.

49. Pattyn N, Cornelissen VA, Eshghi SR, Vanhees $L$. The effect of exercise on the cardiovascular risk factors constituting the metabolic syndrome: a metaanalysis of controlled trials. Sports Med. 2013;43(2):121-33. doi:10.1007/ s40279-012-0003-z

50. Fairey AS, Courneya KS, Field CJ, Bell GJ, Jones LW, Martin BS, et al. Effect of exercise training on C-reactive protein in postmenopausal breast cancer survivors: a randomized controlled trial. Brain Behav Immun. 2005;19(5):381-8. doi:10.1016/j.bbi.2005.04.001

51. Mefferd K, Nichols JF, Pakiz B, Rock CL. A cognitive behavioral therapy intervention to promote weight loss improves body composition and blood lipid profiles among overweight breast cancer survivors. Breast Cancer Res Treat. 2007;104(2):145-52. doi:10.1007/s10549-006-9410-x.

52. Nuri R, Kordi MR, Moghaddasi M, Rahnama N, Damirchi A, Rahmani-Nia F, et al. Effect of combination exercise training on metabolic syndrome parameters in postmenopausal women with breast cancer. J Cancer Res Ther. 2012;8(2):238-42. doi:10.4103/0973-1482.98977.

53. Irwin ML, Varma K, Alvarez-Reeves M, Cadmus L, Wiley A, Chung GG, et al. Randomized controlled trial of aerobic exercise on insulin and insulin-like growth factors in breast cancer survivors: the Yale exercise and survivorship study. Cancer Epidemiol Biomark Prev. 2009;18(1):306-13.

54. Schmitz KH, Ahmed RL, Hannan PJ, Yee D. Safety and efficacy of weight training in recent breast cancer survivors to alter body composition, insulin, and insulin-like growth factor axis proteins. Cancer Epidemiol Biomark Prev. 2005;14(7):1672-80. doi:10.1158/1055-9965.EPI-04-0736.

55. Fairey AS, Courneya KS, Field CJ, Bell GJ, Jones LW, Mackey JR. Effects of exercise training on fasting insulin, insulin resistance, insulin-like growth factors, and insulin-like growth factor binding proteins in postmenopausal breast cancer survivors: a randomized controlled trial. Cancer Epidemiol Biomark Prev. 2003;12(8):721-7.

56. Ross SA, Gulve EA, Wang M. Chemistry and biochemistry of type 2 diabetes. Chem Rev. 2004;104(3):1255-82.

57. Watkinson C, van Sluijs EM, Sutton S, Hardeman W, Corder K, Griffin SJ. Overestimation of physical activity level is associated with lower BMI: a cross-sectional analysis. Int J Behav Nutr Phys Act. 2010;7:68. doi:10.1186/ 1479-5868-7-68

\section{Submit your next manuscript to BioMed Central and we will help you at every step:}

- We accept pre-submission inquiries

- Our selector tool helps you to find the most relevant journal

- We provide round the clock customer support

- Convenient online submission

- Thorough peer review

- Inclusion in PubMed and all major indexing services

- Maximum visibility for your research

Submit your manuscript at www.biomedcentral.com/submit

C Biomed Central 